\title{
System Interconnection as a Path to Bottom up Electrification
}

\author{
Jonathan Bowes \\ University of Strathclyde \\ jonathan.bowes@strath.ac.uk
}

\author{
Campbell Booth \\ University of Strathclyde \\ campbell.d.booth@strath.ac.uk
}

\author{
Scott Strachan \\ University of Strathclyde \\ scott.strachan@strath.ac.uk
}

Electricity access is a key enabling factor for human development, yet over 1.2 billion people have no connection. Minigrids, grid extension and standalone systems are the standard approaches to electrification, but each have significant limitations, including cost of energy, speed of deployment and lack of flexible upgrade paths.

Interconnection of minigrids and Solar Home Systems (SHS) to allow import and export of energy can address many of these issues. Increased diversity can reduce the impacts of variable renewable energy resources and incremental investment over time can facilitate a flexible, bottom up route to universal electrification.

This paper discusses the issues surrounding the existing approaches to electrification in developing countries and outlines the opportunities for minigrid interconnection as a method to accelerate electricity access in a sustainable, cost effective and future-proof manner.

Index Terms-Development, interconnection, renewable energy, smart grid

\section{INTRODUCTION}

Lack of access to electricity is one of the key barriers to human development in much of the world. A strong correlation can be identified between Human Development Index (HDI) and per capita electricity use [1]. Access to clean and affordable energy is one of the UN sustainable development goals [2], and can play a role in facilitating almost all the other goals indirectly. Even basic energy access can improve health, by reducing the volume of harmful indoor pollution from burning solid fuels such as kerosene and provide productive sources of income, communications, information and entertainment.

In this paper, existing practices for electrification will be presented and compared and the potential for another approach involving interconnection of minigrids and SHSs will be explored. Universal electrification by 2030 will require $60 \%$ of those currently without access to be connected by SHSs or minigrids [3]. Benefits of interconnection of these systems will be addressed qualitatively from the perspective of cost, environmental impact and quality of service.

\section{EXISTING APPROACHES}

For the 1.2 billion people without connection to electricity [4], there are currently three routes to electricity access. Each has its own advantages and disadvantages, in terms of cost, quality of service and application. This section will outline the three approaches and consider when each approach is used.

\section{A. Grid Extension}

Extending an existing national grid is the traditional method of connection used in developed countries. It is based on a model of large, centralized generation, usually in the form of thermal power plants, with high voltage transmission moving bulk power across the country and lower voltage distribution networks connecting houses, businesses and industry.

The advantages of this approach are significant: the cheapest cost of installed generation thanks to economies of scale [5] and the largest benefits from diversity of generation and demand. A national grid can usually satisfy high and increasing energy demands from consumers and therefore facilitate demand growth across industrial, domestic and commercial sectors. Reserves of fuels such as coal and natural gas constitute high density energy storage, often removing the need for other storage technologies, such as use of batteries commonplace amongst more distributed topologies utilizing more intermittent renewable energy supplies.

These benefits have resulted in top down, grid connection being the standard method of electrification in most "developed countries".

Unfortunately grid extension and reinforcement is dependent on a number of factors. Firstly, there is a requirement for existing grid infrastructure. Even if infrastructure does exist, it may be unreliable or demand may grow quickly, creating a mismatch, resulting in load shedding and blackouts. [6]

Grid extension is cost effective at connecting densely populated areas, but the more geographically dispersed the population becomes, the greater the cost of distribution network infrastructure per household. Grid extension is more desirable in cities, where large numbers of customers are located close together and in locations close to existing grid infrastructure.

Deployment of grid extension also requires the longest time of the three solutions discussed in this paper. Building large generating plant and transmission networks is labor intensive and requires significant planning. This can leave individuals without access to electricity for extended periods and make investors and governments unwilling to provide interim solutions, such as minigrids due to fear of lost investment and stranded assets in the case of eventual grid extension making a minigrid redundant.

\section{B. Minigrids}

Another approach to electrification is the minigrid. Nomenclature is a confusing issues in this area, with many definitions given for minigrids, microgrids and nanogrids across a wide range of literature, usually referring to the size 
of generation present. There is no clear consensus on the definitions, so for the purpose of this paper, a system featuring generation and distribution but not part of a national grid is considered to be a minigrid, regardless of the size of generation present. Many of the same advantages and issues exist across the range of size from $>1 \mathrm{~kW}$ to multi MW systems.

Minigrids can operate independently from a national grid and many systems are designed to provide energy autonomously to a community in a remote location. Minigrids can also exist connected to a national grid, able to operate autonomously in the case of a blackout in order to ensure a higher system reliability [7].

Minigrids are designed to take advantage of local energy resources, such as solar, hydro and wind.

In recent years, reductions in the cost of solar panels [8] [9] have resulted in the predominant system architecture utilizing a combination of solar photovoltaics, batteries and backup generation.

Minigrids can be implemented more quickly than grid extension, due to the lack of requirement for very large infrastructure projects, such as power stations and transmission networks, but significant planning is still required to understand the demand and generation patterns and costs of the system. Planning tools such as HOMER help system designers to plan the most cost effective solutions, but still require a great deal of knowledge of the local communities and experience to implement effectively.

Minigrids operating mainly on renewable energy require some form of battery storage and backup (diesel) generation to mitigate the inherent variability of the generation technology. This can significantly increase the overall cost of the system. [10].

If a grid connection reaches a location where a minigrid has been deployed, then connecting to the national grid in preference to an existing minigrid may offer better service for customers at a lower cost [11]. In addition, the payback time for a minigrid tends to be many years [12]. Consequently, uncertainty around a national or district electrification plans and strategy can represents a significant risk for investors.

One solution is to construct the minigrid distribution networks to standards compliant with the standards of the local grid operator, making the minigrid capable of interconnecting to the arriving grid extension, and potentially reducing the burden on, and even supporting, centralized utility networks [13]. Many countries are beginning to see the advantages of this approach, with the Nigerian government offering a guarantee to operators of minigrids compliant with their standards to buy the distribution infrastructure from them for its discounted value plus one year's turnover in the case of an expanding grid reaching its location [14].

Another key barrier to the long term success of many minigrids is maintenance and upgrading. Energy use tends to increase over time, but many minigrids are designed for the estimated load at the time of installation, with little consideration of how to react to load growth. This can result in cases where grids suffer regular blackouts [15] as demand continues to grow and the installed minigrid is no longer fit for purpose.

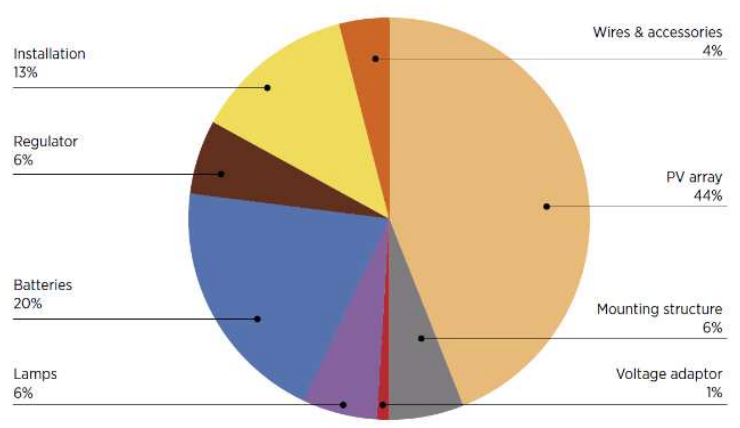

Figure 1: Cost breakdown of $100 \mathrm{Wp}$ solar PV system from [5]

\section{Solar Home Systems}

Solar Home Systems (SHS's) standalone, off-grid solar photovoltaic (PV) systems. They can be considered a subset of minigrids, serving a single house or other load, such as a school or health center. Most consist of a set of solar panels, a charge controller, battery and loads, often operating natively at DC, requiring specialist appliances or the use of an inverter. SHS's are designed to provide basic energy access, such as lighting and phone charging at a relatively low cost. SHSs commonly range in size from $10 \mathrm{Wp}-200 \mathrm{Wp}$ for domestic installations [16] to a few $\mathrm{kW}$ for institutions or productive uses such as community centers, schools, barbers shops, maize mills or irrigations systems.

There has been huge growth in the market for solar home systems over the last few years [14]. In 2016 over 4 million new SHS's found homes in Bangladesh, providing basic electricity access to over 16 million people [5].

One of the key advantages of SHS's are the speed at which they can be deployed. Each system requires no attendant infrastructure to be installed and can be safely fitted with relatively little training due to the inherently safe SELV system voltages (i.e. typically $48 \mathrm{~V}, 24 \mathrm{~V}$ or $12 \mathrm{~V}$ ) [17] and their simple "plug and play" design. Systems can even be redeployed relatively easily to other communities in the case of grid expansion or the installation of a microgrid offering better quality of service, mitigating some of the risk of stranded assets.

SHS's are particularly cost effective in locations with geographically dispersed populations, due to the lack of requirement for distribution infrastructure, but the lack of any benefit from diversity means each individual system must be sized to meet peak demand for the connected loads, resulting in greater installed generation capacity for a given load than either of the other approaches discussed here.

SHSs are even more reliant on storage than minigrids, and require more installed capacity for a given peak load in household, due to the lack of diversity of generation or demand. Figure 1 reflects this, with the cost of an average $100 \mathrm{Wp}$ system dominated by the cost of the PV panels and batteries.

SHS's also tend to be effective mainly due to the low energy demand, most commonly in the order of 10 s of watts for loads such as lighting, phone charging, fans and radios. 


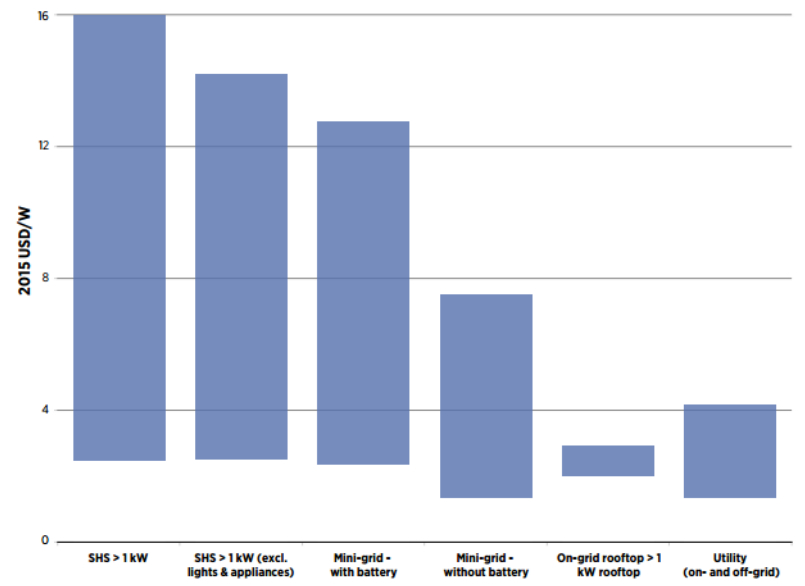

Figure 2: Range of installed costs for solar PV systems in Africa for different types of installations. From [5]

\section{A. Comparison}

With an understanding of the key advantages and disadvantages of each approach, we can begin to examine the "solution space" for electrification.

Grid extension is preferable when there is a geographically dense area, making the attendant infrastructure cost effective. Minigrids are most effective in more remote areas that still have a somewhat dense population and are preferred over the use of SHSs when larger loads are required or when there is significant diversity of demand or generation.

SHSs are preferable in more remote areas with smaller loads and diffuse populations, and where more immediate energy access is required. In practice, they are also used in more densely populated areas in many counties such as Bangladesh, due to low initial cost.

In terms of cost, SHSs require the lease upfront investment, but have the highest levelised cost of energy (LCOE) over their lifetime. Grid extension tends to be the opposite, offering the lowest LCOE, thanks mainly to diversity and economies of scale, as shown in figure 2 , but requiring significant upfront investment, with minigrids occupying a cost middle ground between the two.

These patterns are simply indicative of trends, with the optimal solution being highly contextual. A clear understanding of system requirements, local and cultural considerations and future trends is needed to effectively plan electrification.

\section{INTERCONNECTION}

As discussed above, each approach has clear advantages and disadvantages in differing contexts. There has recently been increasing interests in a different but complimentary method to help achieve electrification, which can mitigate many of the disadvantages of other approaches: interconnection of existing systems.

This approach involves connecting local SHSs or minigrids with a two-way link allowing the transmission of power between the connected systems. Agents could sell excess power onto the market and while suffering a shortage could buy excess energy from neighboring systems, enabling the development of peer-to-peer business models and market dynamics, potentially making energy more affordable to end users.

$30 \%$ of energy in a SHSs is unused due to the requirement to oversize systems to meet the predicted peak demand [18]. If these systems were to be interconnected, some proportion of the excess energy on any given system could be utilized, providing more desired energy to the buyer and a source of income to the seller.

Utilizing interconnection as a planned route to upgrade the electricity infrastructure of a community, area or county can lead to a new approach to electrification, known as bottom up electrification or "swarm electrification".

Rather than the traditional centralized generation, transmission and distribution, requiring significant upfront investment and time, bottom up electrification can support more targeted and incremental investment, which effectively de-risks this market opportunity for potential investors, and so encouraging private sector participation leading to a steady increase in electrification rates.

First, SHSs can be installed to provide basic energy access for lighting, phone charging and other small loads crucial to facilitating human development, utilizing the small upfront cost and quick deployment of SHSs. Over time, if and when demand increases, these systems can be interconnected to increase diversity, share storage, reduce cost of energy and increase reliability. Larger central generation, such as hydro or wind power or back up diesel generation could be added to the created microgrid. Another step up in scale could see a number of microgrids interconnected, further increasing the diversity of generation and demand. Finally, this infrastructure could form a readymade distribution network in the case of grid extension.

The appeal of bottom up electrification is the flexibility it offers, with each stage being optional depending on context. This approach offers the ability to defer upfront investment until there is more certainty of demand.

When interconnecting systems, each of the following benefits must be considered in order to make a cost effective choice.

\section{A. Advantages}

\section{1) Mitigate variability}

Diversity in power system arises from a mismatch between supply or demand caused by differing demand and generation profiles. For a single SHS there is no benefit derived from diversity. The peak demand must be met by the panel and battery at all times to ensure reliable operation. Systems are therefore specified to have large generation and storage.

The more systems connected the greater the benefit from diversity. Additional benefit can be derived from loads with significantly different load profiles. The load profile of a domestic dwelling in a given community will tend to be similar, but schools, healthcare center, small industry, agriculture and may other loads have differing profiles, with peaks at different times. By connecting a variety of loads to the system, each with different operating regimes, diversity can further increase. For example, an industrial application such as 
maize milling could utilize the excess solar energy during the middle of the day, reducing the need for energy storage.

\section{2) Increase reliability}

In the case of a fault on the system, an interconnected network of standalone systems could revert to islanded operation, providing at least limited functionality for basic loads such as lighting, reverting to the original setup of SHSs. This would significantly increase the reliability of systems compared to a minigrid with centralized generation and storage.

A minigrid connected to the national grid could similarly intentionally island its self in the case of a fault on the system The system could also be configured to exhibit reconfiguring or "self-healing" properties, routing power to avoid faults in a similar to the way that the internet can route packets to ensure system performance [19].

There is increasing interest in operating energy systems with the possibility of intentional islanding, even in countries with high electrification rates and established national grids as distributed generation and storage becomes more prevalent across every level of the grid. A bottom up interconnected system could build in this functionality from inception, saving money and effort in the long term.

\section{3) Financial flexibility for individuals}

Many SHSs and minigrids rely on a pay as you go business model or microfinance to fund their operation. Failure to pay is a significant barrier to the commercialization of this technology. The ability to sell excess energy can help individuals or systems to meet payments in difficult times by reducing their electricity demand rather than losing access altogether.

4) Connect new customers with no generation The cost of a solar home system is dominated by the batteries and panel [5] as shown in Figure 1. Individuals who cannot afford to invest in a full SHS could instead install a cheaper controller and converter, connecting their dwelling to a network and allowing them to purchase excess electricity from other connected systems. The quality of service may not be as good as a SHS, as there may be times when all systems of the network are unwilling to sell, but this first step may be an effective method to spread the cost and facilitate quicker and more cost effective electrification for those who cannot afford to purchase a complete SHS. In a location with many SHSs it may make sense for new customers not to buy a SHS at all and simply purchase an interconnection. This could also be used to connect new load centers without energy access to minigrids with excess energy. For example, small enterprises, who don't have the capital to invest in all of the components of an offgrid system could utilize this pooled energy resource and unlock business opportunities within local communities, while also providing income generating potential for prosumers in the sale of energy to these small business.

5) Flexible investment

Investment in the required technology to interconnect systems can be undertaken incrementally, targeting locations with the greatest advantage to be derived, such as those with a varied demand or generation profiles located close together. Initially basic electricity access can be provided quickly and cost effectively with SHSs. There is a documented trend in growth of energy consumption over time as human development increases. As such, this bottom up approach can offer a costeffective method of ensuring the energy infrastructure evolves at a rate in accordance with growing demand. Phased interconnection of SHSs or microgrids can be implemented to gradually increase mini-grid capacity. Additional centralized generation could then be added to the mini-grid and it could eventually be interconnected to other mini-grids, further increasing diversity of demand and supply. Where suitable, this network of mini-grids could then be integrated into a national grid, as and when this becomes possible.

The key advantage is that these upgrades can be undertaken incrementally and can adapt to the changing consumption patterns and need of each community. Though not suitable in every situation, such over large distances or between grids with low diversity, interconnection offers an additional path to grow and upgrade systems over time. In order to fully take advantage of the potential benefits offered by interconnection, a suitable planning methodology is required, involving detailed numerical modelling, real world experience and design tools.

\section{B. Barriers}

1) Standards and compatibility

Well defined and widely adopted standards for voltage levels, connectors, communications protocols and many other factors are crucial to the adoption of interconnection of systems. It is desirable that two systems can be connected as quickly and easily as possible, with the minimum technical expertise required, to reduce costs and allow individuals and communities to have more agency in their own energy.

There are no internationally accepted standards for Low Voltage Direct Current (LVDC), although the IEEE Standards Association is developing a new standard for DC microgrids [20]

\section{2) Distance and diversity}

The value of interconnection is dependent on a number of factors, but the most important are the distance between the potentially connected systems and the level of diversity between them.

$I^{2} R$ losses between two systems are a function of separation distance, as for a given cross-sectional area, the resistance of a line grows linearly with distance. It is often desirable in such systems to use DC voltages less than $48 \mathrm{VDC}$ where possible to ensure touch safety, so resistive losses will be significant. Further investigation is required of the level of diversity and proximity of loads required to make interconnection an effective intervention.

\section{3) Commercialization}

In order to be wildly utilized the technology must not only be positive for consumers, but also financially viable. There are a number of potential business models for interconnection, including PAYG with a centralized pool operated by the 
network owner, Peer to Peer selling with a transaction fee, microfinance or a flat rate subscription.

In order to ascertain the best approach, market research, pilot projects and detailed techno economic modeling are required, with an understanding that there will most likely be a range of suitable solutions depending on the specific social, economic and geographical context.

\section{CONCLUSION}

Access to electricity is a key goal of the UN sustainable development goals and a pathway to improved human development, but all of the existing approaches to electrification present significant shortcomings. The emergence of more heterogeneous network topologies, which are better suited to the bottom-up approach to delivering clean and affordable sources of electricity to the diffuse and remote rural communities of developing countries, offer an the opportunity to leapfrog the traditional concept of top-down, centralized power grid expansion.

There is potential for interconnection of existing systems to mitigate many of the shortcomings, discussed above, facilitating flexible, incremental investment, although there is significant work to be undertaken in modeling and pilot projects to quantify the potential role that interconnection could play in the path to universal electrification.

\section{REFERENCES}

[1] S. Bhattacharyya, "Energy access programmes and sustainable development: A critical review and analysis," Energy for Sustainable Development, vol. 16, no. 3, p. 260-271, 2012.

[2] United Nations, "Transforming our world: the 2030 Agenda for Sustainable Development".

[3] Sustainable Energy for All, "Energy Access Practitioner Network: Towards Achieving Universal Energy Access by 2030," United Nations, 2012.

[4] The International Energy Agency, "Energy Access Database," 2016.

[5] The International Renewable Energy Agency, "Solar PV in Arfica: Costs and Markets," 2016.

[6] N. Onishi, "Weak Power Grids in Africa Stunt Economies and Fire Up Tempers," 2 July 2015. [Online]. Available: https://www.nytimes.com/2015/07/03/world/africa/w eak-power-grids-in-africa-stunt-economies-and-fireup-tempers.html?_r=0. [Accessed 24 May 2017].

[7] S. Conti and S. A. Rizzo, "Monte Carlo Simulation by Using a Systematic Approach to Assess Distribution System Reliability Considering Intentional Islanding," IEEE Transactions on Power Delivery, vol. 30, no. 1, 2014.
[8] T. Srinivasan and D. Rajamani, "Solar Power Renaissance," International Journal of Supply Chain Management, 2016.

[9] R. Fares, "The Price of Solar Is Declining to Unprecedented Lows," Scientific American, 2016.

[10] H. Ibrahim, A. Llinca and J. Perron, "Energy storage systems - Characteristics and comparisons," Renewable and Sustainable Energy Reviews, vol. 12, no. 5, pp. 1221-1250, 2008.

[11] C. Greacen, R. Engel and T. Quetchenbach, "A Guidebook on Grid Interconnection and Islanded Operation of Mini-Grid Power Systems Up to 200kW," Lawrence Berkeley National Laboratory, 2013.

[12] Sustainable Energy For All: Africa Hub, " Green Mini-Grids in Sub-Saharan Africa: Analysis of Barriers to Growth and the Potential Role of the African Development Bank in Supporting the Sector," 2016.

[13] IRENA, "Innovation Outlook Renewable Mini-Grids Summary for Policy Makers," 2016.

[14] Bloomberg New Energy Finance, "Q1 2017 Off-grid and Mini-grid Market Outlook," 2017.

[15] C. W. Shyu, "End-users' experiences with electricity supply from stand-alone mini-grid solar PV power stations in rural areas of western China," Energy for Sustainable Development, vol. 17, no. 4, pp. 391-400, 2013.

[16] The International Renewable Energy Agency, IRENA Renewable Cost Database, 2016.

[17] Sola/Hevi-Duty, "NEC Class 2 Power Circuits and Power Supplies," 2002.

[18] H. Kirchhoff, "Identifying Hidden Resources in Solar Home Systems as the Basis for Bottom-Up Grids," in Innovating Energy Access for Remote Areas: Discovering Untapped Resources, Berkeley, 2014.

[19] Z. Wang, B. Chen, J. Wang and C. Chen, "Networked Microgrids for Self-Healing Power Systems," IEEE Transactions on Smart Grid, vol. 7, no. 1, pp. 310319, 2015.

[20] IEEE, "P2030.10 - Standard for DC Microgrids for Rural and Remote Electricity Access Applications," [Online]. Available: https://standards.ieee.org/develop/project/2030.10.ht ml. [Accessed 25 May 2017].

[21] Sustainable Business Institute, "Scaling up Successful Micro-Utilities for Rural Electrification," 2013. 